ing intracellular bacterial infection. J. Clin. Invest 113:401-413

14. Ehrenstein, M.R., et al. 2004. Compromised function of regulatory $\mathrm{T}$ cells in rheumatoid arthritis and reversal by anti-TNFalpha therapy. J. Exp. Med. 200:277-285

15. Nadkarni, S., Mauri, C., and Ehrenstein, M.R. 2007. Anti-TNF-alpha therapy induces a distinct regulatory $\mathrm{T}$ cell population in patients with rheumatoid arthritis via TGF-beta. J. Exp. Med. 204:33-39.

16. Scott-Browne, J.P., et al. 2007. Expansion and function of Foxp3-expressing $\mathrm{T}$ regulatory cells during tuberculosis. J. Exp. Med. 204:2159-2169.

17. Van den Brande, J.M., et al. 2003. Infliximab but not etanercept induces apoptosis in lamina propria T-lymphocytes from patients with Crohn's disease. Gastroenterology. 124:1774-1785.

18. Lugering, A., et al. 2001. Infliximab induces apoptosis in monocytes from patients with chronic active Crohn's disease by using a caspase-dependent pathway. Gastroenterology. 121:1145-1157.

19. Mitoma, H., et al. 2005. Infliximab induces potent anti-inflammatory responses by outside-to-inside signals through transmembrane TNF-alpha. Gastroenterology. 128:376-392.

20. Mitoma, H., et al. 2008. Mechanisms for cytotoxic effects of anti-tumor necrosis factor agents on transmembrane tumor necrosis factor alpha-expressing cells: comparison among infliximab, etanercept, and adalimumab. Arthritis Rheum. 58:1248-1257.

21. Scallon, B.J., Moore, M.A., Trinh, H., Knight, D.M., and Ghrayeb, J. 1995. Chimeric anti-TNF-alpha monoclonal antibody cA2 binds recombinant transmembrane TNF-alpha and activates immune effector functions. Cytokine. 7:251-259.

22. Sandborn, W.J., et al. 2001. Etanercept for active Crohn's disease: a randomized, double-blind, placebo-controlled trial. Gastroenterology. 121:1088-1094.

23. Dinarello, C.A. 2005. Differences between antitumor necrosis factor-alpha monoclonal antibodies and soluble TNF receptors in host defense impairment. J. Rheumatol. Suppl. 74:40-47.

\title{
CaMKII and a failing strategy for growth in heart
}

\author{
Mark E. Anderson
}

Department of Internal Medicine, Division of Cardiovascular Medicine, and Department of Molecular Physiology and Biophysics, University of lowa Carver College of Medicine, lowa City, lowa, USA.

\begin{abstract}
Patients with systolic left ventricular dysfunction die progressively from congestive heart failure or die suddenly from cardiac arrhythmias. Myocardial hypertrophy is an early event in most forms of heart failure, but the majority of patients with myocardial hypertrophy do not develop heart failure. Developing improved therapies for targeting the cell signaling pathways that enable this deadly transition from early myocardial insult to heart failure and sudden death is a key goal for improving public health. In this issue of the JCI, Ling and colleagues provide new evidence that activation of the multifunctional $\mathrm{Ca}^{2+} /$ calmodulin-dependent kinase II $\delta$ is a decisive step on the path to heart failure in mice (see the related article beginning on page 1230).
\end{abstract}

\section{The heart failure epidemic}

Heart failure is a leading cause of death in the developed world, and the incidence of heart failure continues to rise despite impressive improvements in treating coronary artery disease and hypertension. Heart failure patients encounter 2 main problems: reduced cardiac pumping that is inadequate to meet metabolic demands, and electrical instability that causes arrhythmias and sudden death. Heart failure is a clinical syndrome, but most heart failure in the developed world is caused by reduced left ventricular systolic function as a result of hypertension and myocardial infarction. Antihypertensive medications have resulted in a reduction in the number

Conflict of interest: Mark E. Anderson is named as the inventor on patents issued and pending regarding the treatment of heart failure and arrhythmias by CaMKII inhibition.

Nonstandard abbreviations used: $\mathrm{Ca}^{2+} / \mathrm{CaM}$, calcified calmodulin; CaMKII, $\mathrm{Ca}^{2+} /$ calmodulin-dependent kinase II; HDAC, class II histone deacetylase; MEF2, myocyte enhancer factor 2 .

Citation for this article: J. Clin. Invest. 119:1082-1085 (2009). doi:10.1172/JCI39262. of patients with myocardial hypertrophy (1), and improved treatment of myocardial infarction patients has drastically reduced the acute and 5-year mortality of this common form of structural heart disease (2). Unfortunately, the incidence of heart failure has increased, despite reducing the burden of hypertension and the lethality of myocardial infarction (2). Improved understanding of myocardial biology favoring conversion of early forms of structural heart disease into the advanced syndrome of heart failure and sudden death is needed to devise more effective approaches to preventing and treating heart failure.

\section{Why is CaMKII important in myocardial disease?}

Many signaling molecules are implicated in structural heart disease. However, few, if any, molecules appear to be as central to pathological response mechanisms in heart as $\mathrm{Ca}^{2+} /$ calmodulin-dependent kinase II (CaMKII). CaMKII expression and activity are increased in failing human myocardium and in many animal models of cardiac hypertrophy and heart failure. CaMKII overexpression in mouse myocardium causes hypertrophy, failure, and sudden death (3), while CaMKII inhibition suppresses these phenotypes $(4,5)$. Pathological stress induces a cardiomyocyte milieu of dysregulated and prolonged cytoplasmic $\mathrm{Ca}^{2+}$ transients (6) and increased oxidative stress (7). CaMKII is an ideal nodal molecule for transducing $\mathrm{Ca}^{2+}$ and redox signals fundamental upstream signals common to most forms of structural heart disease into downstream events, such as apoptosis (8), hypertrophy (3), and proarrhythmic electrical remodeling $(3,4)$, that lead to the clinical phenotypes of congestive heart failure and sudden death (Figure 1A). Finally, CaMKII itself amplifies the disruption of intracellular $\mathrm{Ca}^{2+}$ homeostasis in heart failure by increasing the opening probability of voltage-gated $\mathrm{Ca}^{2+}$ channels (9) and ryanodine receptors, the latter being the major mediators of $\mathrm{Ca}^{2+}$-induced $\mathrm{Ca}^{2+}$ release from intracellular sarcoplasmic reticulum $\mathrm{Ca}^{2+}$ stores in cardiac myocytes (3).

CaMKII is initially activated by binding to calcified calmodulin $\left(\mathrm{Ca}^{2+} / \mathrm{CaM}\right) . \mathrm{Ca}^{2+} / \mathrm{CaM}$ binding reorders the structure of the CaMKII molecule by disinhibiting the catalytic domain and exposing the regulatory domain (Figure 1B). The CaMKII regulatory domain is the target for activity-sustaining modifications that bring about the transition of CaMKII into a $\mathrm{Ca}^{2+} / \mathrm{CaM}$-autonomous enzyme. $\mathrm{Ca}^{2+} / \mathrm{CaM}$-activated CaMKII monomers are susceptible to autophosphorylation (10) at threonine $286 / 287$ (the specific number is isoform dependent) and oxidation of paired methionines 281 and 282 (8). CaMKII autophosphorylation and oxidation prevent 
A

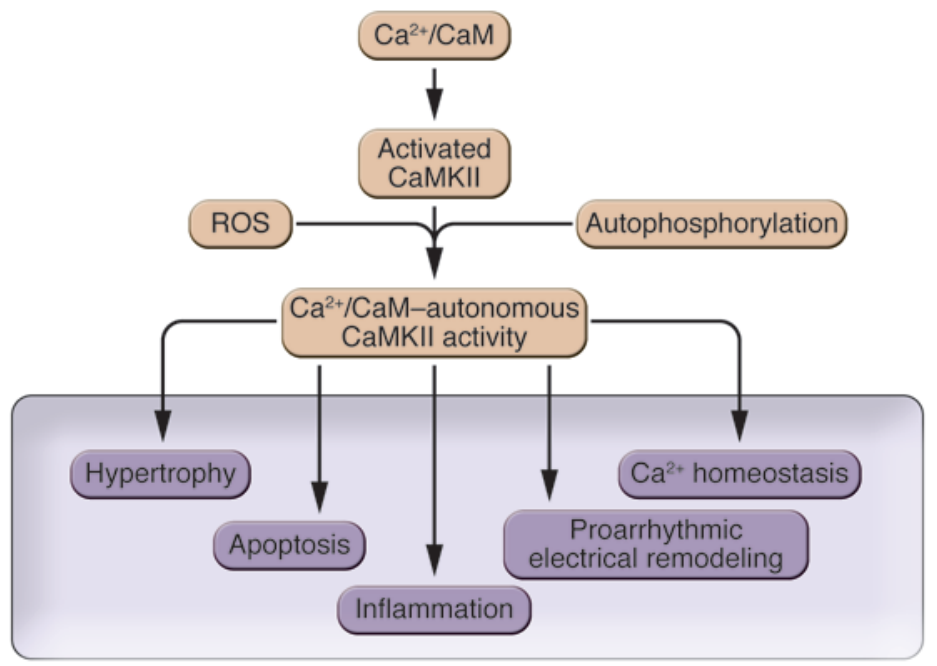

B

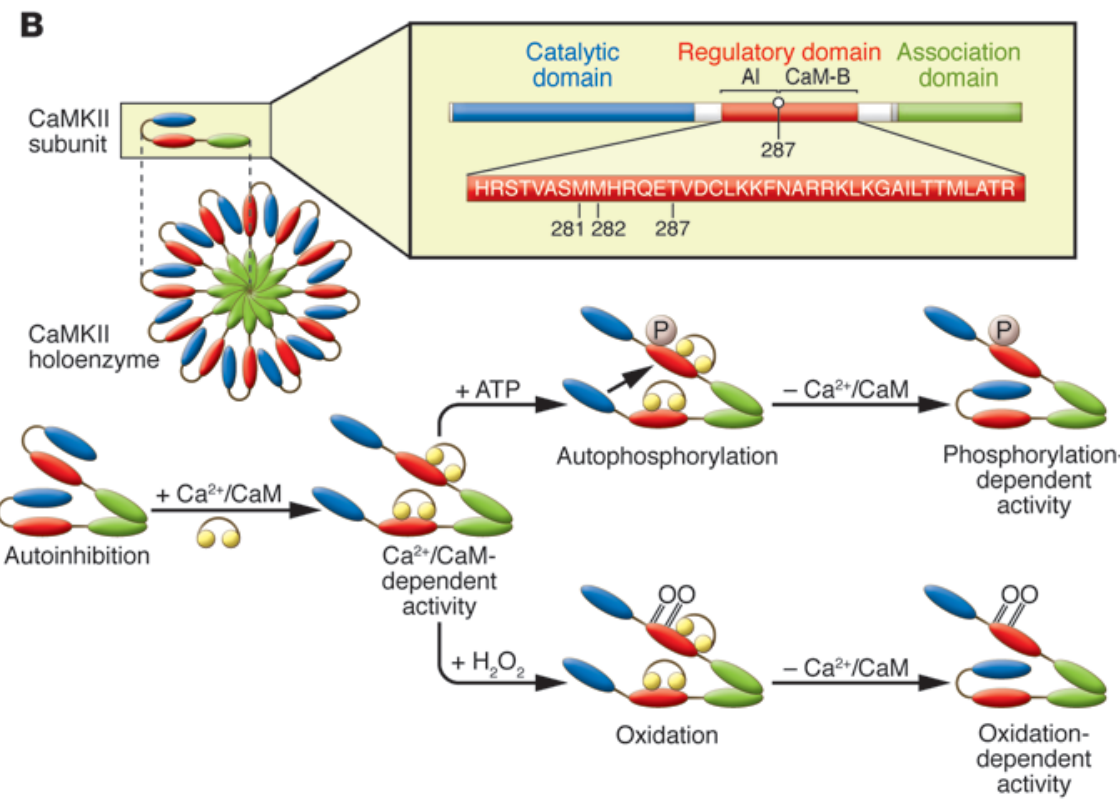

Figure 1

CaMKII activation is initiated by $\mathrm{Ca}^{2+} / \mathrm{CaM}$, sustained by autophosphorylation and oxidation, and leads to downstream cellular events important for the development of heart failure. (A) $\mathrm{Ca}^{2+} / \mathrm{CaM}$-activated CaMKII transitions to $\mathrm{Ca}^{2+} / \mathrm{CaM}$-autonomous activity by exposure to ROS or through autophosphorylation. Excessive CaMKII activity triggers diverse, maladaptive cellular events important for the transition from early myocardial stress to late-stage myocardial diseases of heart failure, arrhythmias, and sudden death. (B) Top: The CaMKII holoenzyme and a single CaMKII subunit. The regulatory domain consists of an autoinhibitory region (Al) and a calmodulin-binding region (CaM-B). Bottom: CaMKII becomes constitutively active by autophosphorylation $(\mathrm{P})$ and/or oxidation $(\mathrm{O})$.

inhibition of the CaMKII catalytic domain by the autoinhibitory region within the regulatory domain, even after reversal of $\mathrm{Ca}^{2+} /$ CaM binding. Thus, loss of cytoplasmic $\mathrm{Ca}^{2+}$ homeostasis and increased oxidation, ubiquitous responses of pathologically stressed myocardium, both activate CaMKII and convert CaMKII from a $\mathrm{Ca}^{2+}$-dependent to a $\mathrm{Ca}^{2+}$-independent signaling molecule.
CaMKII is multifunctional. Excessive CaMKII activates hypertrophic genes, at least in part, by phosphorylating class II histone deacetylases (HDACs) and by derepressing myocyte enhancer factor 2-dependent (MEF2-dependent) transcription (11). CaMKII also increases activity of NF- $\mathrm{KB}$ to activate inflammatory response genes after myocardial infarction (12). CaMKII actions are not confined to regulation of transcriptional pathways. CaMKII participates in the loss of intracellular $\mathrm{Ca}^{2+}$ homeostasis and proarrhythmic electrical remodeling (3, 4) that are consistent features of heart failure, and the actions of CaMKII on voltage-gated $\mathrm{Ca}^{2+}$ channels (9) and $\mathrm{Na}^{+}$ channels (13) are important components of the proarrhythmic actions of CaMKII. CaMKII triggers sarcoplasmic reticulum $\mathrm{Ca}^{2+}$ leak in heart failure (14), which may be an early contributor to membrane potential instability and arrhythmias. CaMKII is a proapoptotic signal during myocardial infarction (15), excessive catecholamine (16) and angiotensin II (8) exposure, and aortic banding surgery (17). Thus, CaMKII is a hypertrophic signaling molecule, but it participates in other cellular events that may be critical for the transition from early structural heart disease to heart failure and sudden death.

\section{One mouse, two phenotypes?}

There are 4 CaMKII isoforms (CaMKII $\alpha$, CaMKII $\beta$, CaMKII $\gamma$, and CaMKIII), but the $\delta$ and $\gamma$ isoforms appear to predominate in myocardium. Two leading groups recently reported on new Camk2 $d^{-/-}$mouse models. Backs et al. targeted Camk2d exons 1 and 2, which encode the ATP-binding domain (18), while Ling et al., in their study in this issue of the JCI, targeted exons 9-11, which encode the catalytic domain (17). Both groups provided convincing evidence that CaMKIId expression was eliminated, and both groups found that under basal conditions, Camk2 $d^{-/-}$mice had structurally and functionally normal hearts. Both groups selected an aortic banding model of pathological afterload, a condition demanding increased work to expel blood from the left ventricle, as an initial test of the effect of eliminating CaMKIIס expression. Aortic banding resembles acute-onset severe aortic valve stenosis. Ling et al. found that Camk2d $d^{-/}$and WT littermate controls had similar myocardial hypertrophy responses at 2 weeks, an early time point in the aortic banding, but that prolonged banding for 6 weeks resulted in marked left ventricular dilation and heart failure only in WT mice (17). In contrast, Backs et al. found that their Camk2 $d^{-/-}$mice were resistant to myocardial hypertrophy after 3 weeks of aortic banding compared with WT animals, but they did not report on whole heart structure or function at a later time point (18). However, ventricular myocytes isolated 
6 weeks after aortic banding in the study by Backs et al. contracted normally and showed no change in cytoplasmic $\mathrm{Ca}^{2+}$ handling. In the Ling et al. study, ventricular myocytes isolated from WT mice 6 weeks after aortic banding showed increased ryanodine receptor $\mathrm{Ca}^{2+}$ leak compared with ventricular myocytes isolated from Camk2 $d^{-/-}$mice (17). The findings of both groups suggested that alternative Camk2 genes are upregulated in Camk2 $d^{-/-}$mice. Backs et al. found no change in the expression of the CaMKII association domain, the sequence of which is nearly identical in both CaMKIII and CaMKII $\gamma$, despite elimination of the CaMKIIS protein from these animals (18). Conversely, Ling et al. found significant and equivalent increases in total autophosphorylated CaMKII in hearts from Camk2 $d^{-/-}$and WT mice 2 weeks after aortic banding (17). Notably, Ling et al. found increased expression and activity (detected as autophosphorylation) of PKD in both WT and Camk2 $d^{-/-}$mice 2 weeks after aortic banding. PKD is a CaMKII family protein that is not directly activated by $\mathrm{Ca}^{2+} / \mathrm{CaM}$, but shares phosphorylation consensus sites with CaMKII and is known to participate in HDAC- and MEF2-mediated cardiac hypertrophy signaling (19).

While it is presently unclear why the studies of Backs et al. and Ling et al. (17, 18) appear to show conflicting results, it is easy to posit testable reasons for these differences. Aortic banding surgery is technically challenging, and minute differences in the degree of constriction result in major outcome differences: from no overt effect, to hypertrophy, to heart failure, to immediate perioperative mortality. Thus, it is possible that aortic banding was tighter in the animals studied by Ling et al. than in those studied by Backs et al. Other occult differences in the mouse models, such as differences in the genetic backgrounds, age, and gender mix, could also be important. In my opinion, these differences should not mask a key finding shared by both studies: that CaMKIId is important for transduction of myocardial stress responses into clinically relevant disease phenotypes.

\section{One hit for hypertrophy, multiple hits for heart failure}

It is absolutely clear that myocardial hypertrophy is an independent risk factor for decreased left ventricular function and heart failure. However, most patients with myocardial hypertrophy do not transition to reduced systolic function or heart failure.
The Cardiovascular Health Study followed 1,092 patients with increased left ventricular mass and the same number of patients, matched for age and ethnicity, without increased left ventricular mass (20). At the time of enrollment, both patient groups had normal left ventricular systolic function, as measured by echocardiography. After 5 years, only $12.3 \%$ of patients in the highest quartile of left ventricular mass at enrollment developed any detectable left ventricular systolic function, and $6.9 \%$ of these patients developed clinical heart failure. Thus, left ventricular hypertrophy is an important risk factor, but falls short of being a decisive event for causing systolic dysfunction and congestive heart failure.

It is increasingly clear that activation of concise transcriptional signaling pathways can cause cardiac hypertrophy, while the syndrome of systolic dysfunction and congestive heart failure is more complex. Some of the important factors that independently favor transition from myocardial hypertrophy to reduced systolic function and congestive heart failure in patients are hypertension, ischemia, myocardial infarction, diabetes, and valvular heart disease (21). Excessive activity of circulating neurohumoral signals causes experimental heart failure, and increased circulating norepinephrine (22) and renin (23) correlate with increased mortality in patients. These clinical associations suggest, but do not prove, that heart failure is the result of multiple pathological hits, likely involving cellular mechanisms not directly linked to hypertrophy.

\section{CaMKII inhibition as a therapy for experimental heart failure}

CaMKII is an appealing therapeutic target in heart disease, not only because CaMKII inhibition reduces cardiac hypertrophy, but because CaMKII inhibition - by Camk2d knockout (17), transgenic overexpression of inhibitory peptides mimicking the CaMKII autoinhibitory region (Figure 1B), or small molecules (4) - protects against heart failure, at least in mice. CaMKII inhibition, by expression of inhibitory peptides that target all CaMKII isoforms in cardiac myocytes, lessens adverse remodeling after myocardial infarction (4), improves myocardial function, and reduces mortality in calcineurin-mediated cardiomyopathy (5) without affecting myocardial hypertrophy. Thus, CaMKII inhibition can improve a failing heart without reducing myocardial hypertrophy. The benefits of CaMKII inhibition in mouse models of heart failure are most likely caused by the multiple pathological events orchestrated by CaMKII activation: hypertrophy, apoptosis, inflammation, loss of $\mathrm{Ca}^{2+}$ homeostasis, and proarrhythmic electrical remodeling (Figure 1A). The disease resistance seen in genetic models in which CaMKII was inhibited by a peptide inhibitor $(4,5,8$, 12) could in principle be caused, at least in part, by inhibitory actions on PKD. However, the finding by Ling et al. that PKD expression and activation were significantly and equivalently increased in both Camk2d $d^{-/}$and WT mice with incipient heart failure (17) suggests that the effects of PKD are not critical for the transition from hypertrophy to heart failure, at least in response to aortic banding. The exciting studies by Ling et al. and Backs et al. $(17,18)$ add to evidence that CaMKII is a molecule to watch in structural heart disease and provide hope that new CaMKIIbased therapeutics could reduce suffering in patients with heart failure.

\section{Acknowledgments}

This work was funded by NIH R01 grants HL 079031, HL 62494, and HL 70250; by the University of Iowa Research Foundation; and by the Fondation Leducq Alliance for CaMKII signaling.

Address correspondence to: Mark E. Anderson, Department of Internal Medicine, Division of Cardiovascular Medicine, and Department of Molecular Physiology and Biophysics, University of Iowa, Iowa City, Iowa 52242, USA. Phone: (319) 3537107; Fax: (319) 353-6343; E-mail: mark-eanderson@uiowa.edu.

1. Mosterd, A., et al. 1999. Trends in the prevalence of hypertension, antihypertensive therapy, and left ventricular hypertrophy from 1950 to 1989 . N. Engl. J. Med. 340:1221-1227.

2. Velagaleti, R.S., et al. 2008. Long-term trends in the incidence of heart failure after myocardial infarction. Circulation. 118:2057-2062.

3. Zhang, T., et al. 2003. The deltaC isoform of CaMKII is activated in cardiac hypertrophy and induces dilated cardiomyopathy and heart failure. Circ. Res. 92:912-919.

4. Zhang, R., et al. 2005. Calmodulin kinase II inhibition protects against structural heart disease. Nat. Med. 11:409-417.

5. Khoo, M.S., et al. 2006. Death, cardiac dysfunction, and arrhythmias are increased by calmodulin kinase II in calcineurin cardiomyopathy. Circulation. 114:1352-1359.

6. Gwathmey, J.K., et al. 1987. Abnormal intracellular calcium handling in myocardium from patients with end-stage heart failure. Circ. Res. 61:70-76.

7. Maack, C., et al. 2003. Oxygen free radical release in human failing myocardium is associated with increased activity of rac1-GTPase and represents a tar- 
get for statin treatment. Circulation. 108:1567-1574.

8. Erickson, J.R., et al. 2008. A dynamic pathway for calcium-independent activation of CaMKII by methionine oxidation. Cell. 133:462-474.

9. Dzhura, I., Wu, Y., Colbran, R.J., Balser, J.R., and Anderson, M.E. 2000. Calmodulin kinase determines calcium-dependent facilitation of L-type calcium channels. Nat. Cell Biol. 2:173-177.

10. Schworer, C.M., Colbran, R.J., and Soderling, T.R 1986. Reversible generation of a Ca2+-independent form of $\mathrm{Ca} 2+($ calmodulin)-dependent protein kinase II by an autophosphorylation mechanism. J. Biol. Chem. 261:8581-8584.

11. Lu,J.,McKinsey, T.A., Nicol, R.L., and Olson, E.N. 2000 Signal-dependent activation of the MEF2 transcription factor by dissociation from histone deacetylases. Proc. Natl. Acad. Sci. U. S. A. 97:4070-4075.

12. Singh, M.V., et al. 2009. $\mathrm{Ca}^{2+} /$ calmodulin-dependent kinase II triggers cell membrane injury by inducing complement factor $\mathrm{B}$ gene expression in the mouse heart. J. Clin. Invest. 119:986-996.
13. Wagner, S., et al. 2006. Ca2+/calmodulin-dependent protein kinase II regulates cardiac $\mathrm{Na}+$ channels. J. Clin. Invest. 116:3127-3138.

14. Ai, X., Curran, J.W., Shannon, T.R., Bers, D.M., and Pogwizd, S.M. 2005. Ca2+/calmodulin-dependent protein kinase modulates cardiac ryanodine receptor phosphorylation and sarcoplasmic reticulum Ca2+ leak in heart failure. Circ. Res. 97:1314-1322.

15. Yang, Y., et al. 2006. Calmodulin kinase II inhibition protects against myocardial cell apoptosis in vivo. Am. J. Physiol. Heart Circ. Physiol. 291:H3065-H3075.

16. Zhu, W.Z., et al. 2003. Linkage of beta(1)-adrenergic stimulation to apoptotic heart cell death through protein kinase A-independent activation of $\mathrm{Ca}^{2+}$ calmodulin kinase II. J. Clin. Invest. 111:617-625.

17. Ling, H., et al. 2009. Requirement for $\mathrm{Ca}^{2+} /$ calmodulin-dependent kinase II in the transition from pressure overload-induced cardiac hypertrophy to heart failure in mice. J. Clin. Invest. 119:1230-1240.

18. Backs, J., et al. 2009. The delta isoform of CaM kinase II is required for pathological cardiac hyper- trophy and remodeling after pressure overload. Proc. Natl. Acad. Sci. U. S. A. 106:2342-2347.

19. Vega, R.B., et al. 2004. Protein kinases C and D mediate agonist-dependent cardiac hypertrophy through nuclear export of histone deacetylase 5 . Mol. Cell. Biol. 24:8374-8385.

20. Drazner, M.H., et al. 2004. Increased left ventricular mass is a risk factor for the development of a depressed left ventricular ejection fraction within five years: The Cardiovascular Health Study. J. Am. Coll. Cardiol. 43:2207-2215.

21. Levy, D., Larson, M.G., Vasan, R.S., Kannel, W.B., and Ho, K.K. 1996. The progression from hypertension to congestive heart failure. JAMA. 275:1557-1562.

22. Benedict, C.R., et al. 1996. Prognostic significance of plasma norepinephrine in patients with asymptomatic left ventricular dysfunction. SOLVD Investigators. Circulation. 94:690-697.

23. Parikh, N.I., et al. 2007. Plasma renin and risk of cardiovascular disease and mortality: The Framingham Heart Study. Eur. Heart J. 28:2644-2652.

\title{
Genetic susceptibility to HIV-associated nephropathy
}

\author{
Susan E. Quaggin
}

Division of Nephrology, St. Michael's Hospital, and Samuel Lunenfeld Research Institute, Mount Sinai Hospital, University of Toronto, Toronto, Ontario, Canada.

HIV-1-associated nephropathy (HIVAN) is a common complication of HIV-1 infection, and its skewed incidence in certain ethnic groups suggests that there is a genetic basis to HIVAN susceptibility. In their study reported in this issue of the JCI, Papeta and colleagues used a combination of gene expression profiling and linkage analysis to identify three genomic loci that regulate a network of genes expressed by podocytes - cells that are crucial to the filtration of fluid and waste by the kidney (see the related article beginning on page 1178). Surprisingly, two of these loci confer disease susceptibility in a transgenic mouse model of HIVAN. This report confirms the central role of podocytes in the pathogenesis of HIVAN and demonstrates the power of this combination of genomic analysis techniques in elucidating the pathogenesis of glomerular disease.

HIV-1-associated nephropathy (HIVAN) is a leading cause of morbidity and mortality in HIV-1-infected patients. There is a significant component of genetic risk in HIVAN, as it occurs almost exclusively in patients of African descent (1). Histologically, the renal lesion is characterized by collapsing glomerulopathy, epithelial cell proliferation, tubuloreticular inclusions, microcystic dilation of the tubules, and tubulointerstitial inflammation (reviewed in refs. 2,3). Over the past decade, much

Conflict of interest: The author has declared that no conflict of interest exists.

Nonstandard abbreviations used: eQTL, expression quantitative trait locus; HIVAN, HIV-1-associated nephropathy; Myh9, myosin, heavy chain 9, non-muscle; Nphs1, nephrosis 1 homolog; Synpo, synaptopodin. Citation for this article: J. Clin. Invest. 119:1085-1089 (2009). doi:10.1172/JCI39254. has been learned about the pathogenesis of HIVAN at a cellular level (reviewed in ref. 2). Although the involvement of tubular epithelial cells, endothelial cells, and T cells has been reported, emerging data have supported a major role for the podocyte in the development of HIVAN (4-6).

\section{Podocytes and the glomerular filtration barrier}

Podocytes (also known as glomerular visceral epithelial cells) are unusual cells that are only found in the renal glomerulus. Together with glomerular endothelial cells and the intervening glomerular basement membrane, they form the glomerular filtration barrier, a permselective sieve and the site of formation of the primary urinary filtrate (7) (Figure 1). The identification of mutations in genes that are expressed by podocytes has explained the cause of a number of Mendelian glomerular diseases (8-13) and has placed the podocyte in a central position in studies of glomerular disease. In patients with HIVAN, direct HIV-1 infection of podocytes has been documented, and viral proteins induce podocyte proliferation, loss of cell contact inhibition, and upregulation of growth factors in immortalized podocytes in culture (14-17). The concept of a dysregulated podocyte phenotype in HIVAN has been proposed (15), and this phenotype is characterized by loss of expression of many genes typically seen in differentiated podocytes, such as synaptopodin (SYNPO), nephrosis 1 homolog (NPHS1; which encodes the slit-diaphragm protein nephrin), and NPHS2 (which encodes the podocyte slit diaphragm protein podocin), and with the re-expression of embryonic genes such as paired box gene 2 (PAX2) that are normally only expressed in presumptive podocytes and podocyte progenitors during glomerular development.

\section{Genetic susceptibility and HIV}

In North America, HIVAN is largely restricted to patients of African descent, suggesting that genetic factors are important in the development of or susceptibility to this disease. Furthermore, the relative risk for developing end-stage renal disease is 